\title{
A Modern Method to Improve of Detecting and Categorizing Mechanism for Micro Seismic Events Data Using Boost Learning System
}

\author{
Saeed Ghorbani ${ }^{\mathrm{a}}{ }^{*}$, Morteza Barari ${ }^{\mathrm{b}}$, Mojtaba Hosseini ${ }^{\mathrm{c}}$ \\ ${ }^{a}$ Ph.D. Student, Malek-Ashtar University of Technology, Faculty of Information and Communication Technology, Tehran, Iran. \\ ${ }^{b}$ Professor, Malek-Ashtar University of Technology, Faculty of Information and Communication Technology, Tehran, Iran. \\ ${ }^{c}$ Assistant Professor, Malek-Ashtar University of Technology, Faculty of Information and Communication Technology, Tehran, Iran.
}

Received 31 July 2017; Accepted 25 September 2017

\begin{abstract}
Various natural disasters such as floods, fires, earthquakes, etc. have affected human life. Detection and classification of large and small earthquakes caused by natural or abnormal events have been always important to Earth scientist. One of the most important research challenges in this field is the lack of an effective method for identifying and categorizing various types of seismic events at less important and important levels. Based on latest achievements of Data Mining international institutions such as Rexer-KDnugget-Gartner and also newest authentic articles, SVM, KNN, C4.5, MLP are from most important and popular and leading classifiers in data world. Therefor in present study, a boost learning system consisting support vector machine algorithms with linear regression, MLP Neural Network 'C4.5 decision tree and KNN near neighbourhood have been utilized in a combined form to detect and categorize micro seismic events. In general, the steps involved in the proposed method are: 1) performing artificial seismic tests, 2) data gathering and analysis, 3) conducting pre-processing and separating training and testing samples, 4) generating relevant models with training samples and detecting and clustering test samples and 5) extracting a cluster with the maximum candidate using boost learning. After simulations, it was observed that the accuracy of proposed boost method to the best answer was about $6.1 \%$ higher compare to other methods and the error rate was $0.082 \%$ of recalling. Accuracy of detection and classification to the best answer were also improved compare to other methods up to $2.31 \%$ and $6.34 \%$, respectively.
\end{abstract}

Keywords: Seismic Events; Seismic Data Classification; Boost Learning; Micro Seismic Detection.

\section{Introduction}

People face a variety of natural disasters in their life such as earthquakes, floods, fires and volcanoes from the past until now [1,2]. These disasters have impacted human life and imposed irreparable damage on them. Micro seismic monitoring is the passive observation of very small-scale earthquakes which occur in the ground as a result of human activities or industrial processes such as mining, hydraulic fracturing, enhanced oil recovery, geothermal operations or underground gas storage. Micro seismic science grew out of earthquake seismology and focuses on micro-earthquakes (i.e. magnitude less than zero). These micro-earthquakes are too small to be felt on the surface, but they can be detected by sensitive equipment such as geophones and accelerometers. Various methods are used to forecast the occurrence of earthquake events such as mathematical modeling, ionosphere analysis and studying animals' behavior [3,5]. Some these methods only utilize from a single feature and are not able to use different features in earthquake forecasting. Therefore,

\footnotetext{
* Corresponding author: saeed.ghorbani@gmail.com
}

DOI: http://dx.doi.org/10.21859/cej-03098

> This is an open access article under the CC-BY license (https://creativecommons.org/licenses/by/4.0/).

(C) Authors retain all copyrights. 
earthquake events cannot be correctly forecasted through analyzing only a feature [6]. Nowadays, methods related to computer science can play important roles in different felids. Data mining techniques [7] and machine learning techniques [8] can be very useful in detection and classifying micro seismic events. In the other side, boost learning is being commonly used in many applications related to data mining including forecast, detection and classification and so on and is very effective in providing optimum and desirable output. Hence in present study, micro seismic events have been detected and then classified by combining machine learning techniques including support vector machine with linear regression [9, 10], MLP Neural Network [11] and C4.5 decision tree and (Ross Quinlan, 2014) [12] KNN [13]. Near neighborhood. The rest of present study is as follow: the literature review of study has been provided in section 2. Section (3) presents the suggested model with a description proposed architecture. In sections (4) and (5) the results have been represented and section (6) has been allocated to conclusion and future recommendations.

\section{Literature Review}

\subsection{Micro Seismicity}

Large-scale earthquakes are caused when energy is released as a result of rock failure along a fault. In contrast, micro seismic events are caused when human activities such as mining or oil and gas production change the stress distribution or the volume of a rock mass. When the rock attempts to redistribute the stress within the rock mass, it will suddenly slip or shear along pre-existing zones of weakness such as along faults or fracture networks Traditional micro seismic mapping determines the location and magnitude of the event. When micro seismicity is observed over time, operators may start to see patterns of seismicity related to production activities. Advanced micro seismic analysis performed by ESG can reveal more detailed information about the micro seismicity of the area and how the rock is responding to mining or oil and gas production activities, leading to increased efficiency and optimized operations. This small failure results in the release of energy in the form of seismic waves and is known as a micro seismic event. Passive micro seismic data are commonly buried in noise, which presents a significant challenge for signal detection and recovery. For recordings from a surface sensor array where each trace contains a time-delayed arrival from the event, we propose an autocorrelation-based stacking method that designs a denoising filter from all the traces, as well as a multi-channel detection scheme. This approach circumvents the issue of time aligning the traces prior to stacking because every trace's autocorrelation is centered at zero in the lag domain. The effect of white noise is concentrated near zero lag, so the filter design requires a predictable adjustment of the zero-lag value. Truncation of the autocorrelation is employed to smooth the impulse response of the denoising filter. In order to extend the applicability of the algorithm, we also propose a noise pre-whitening scheme that addresses cases with colored noise. The simplicity and robustness of this method are validated with synthetic and real seismic traces.

In the earthquake-seismology community, a micro seismic event typically is defined as an earthquake that is not "felt" by the public, which usually implies an earthquake with a "moment magnitude" Mw less than about 3 or 4 . Mw is a common measure of an earthquake's strength and is a dimensionless quantity defined as $\mathrm{Mw}=0.67 \log 10(\mathrm{M} 0)-6.07$. In the equation above, the variable M0 is the "seismic moment" in units of [N•m], which is a quantitative measure of the amount of energy released in an earthquake such that $\mathrm{M} 0=\mu \mathrm{A} D$, where $\mathrm{A}$ is the surface area of the rupture created by the earthquake along a fault or fracture, $\mathrm{D}$ is the amount of rock displacement along the rupture surface, and $\mu$ is the average shear strength of the rock encompassing the ruptured zone. A fracture is defined as a crack or break in a rock, and a fault is defined as a fracture along which relative displacement or "throw" of the rock has occurred across the fracture. For typical fluid injection or withdrawal scenarios, reservoir pressure changes of a few hundred psi (a few megapascals) can induce weak micro seismic events of magnitudes less than $\mathbf{M}-2$, with associated rock displacements much less than a millimeter along fracture scale lengths much shorter than a meter. An M-2 event is approximately one billion times weaker (less energetic) than earthquakes that the public can feel. Moderate "fault reactivation" or significant "rock-fracturing" events typically induce micro seismic energy of magnitudes less than M2, with associated rock displacements less than a centimeter along fracture or fault lengths shorter than a few (or tens of) meters. An M2 event is approximately 100 to 1000 times weaker than what the public can feel. Large fault related earthquake rupture events typically radiate seismic energy with magnitudes greater than M4, with associated fault slip displacements greater than several centimeters or meters along fault lengths greater than several (tens or hundreds of) kilometers. An M3 or M4 event is typically the weakest magnitude that the public can feel. 


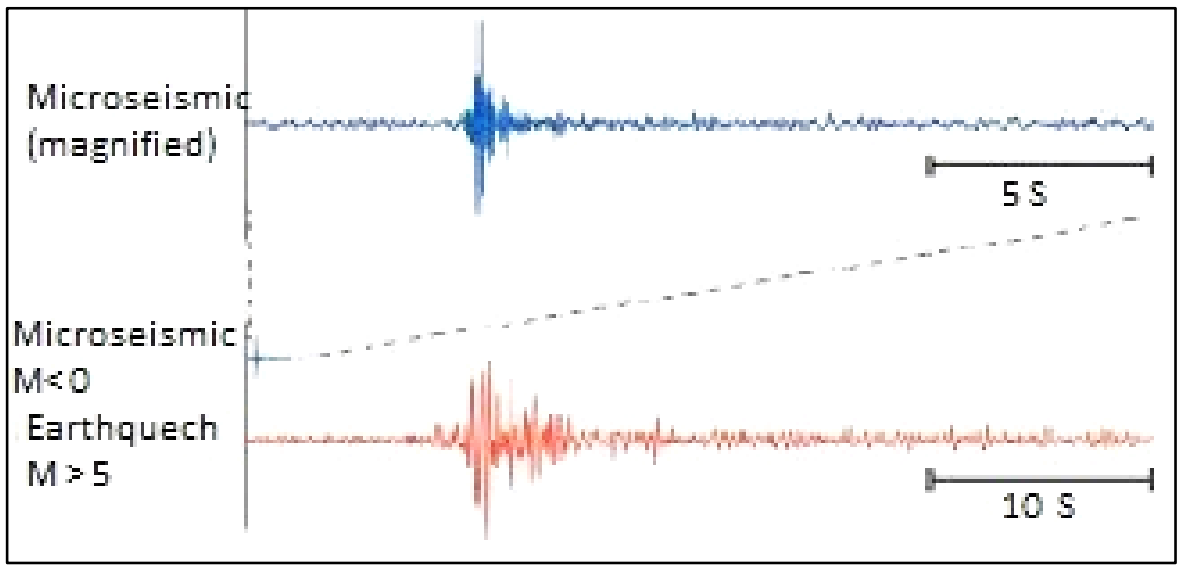

Figure 1. Example of recorded seismic waveforms generated by an $\mathrm{Mw}<0$ induced micro seismic event (blue) and an $\mathrm{Mw}>$ 5 natural felt earthquake (red)

Figure 1. compares an induced micro seismic waveform $(\mathrm{M}<0)$ with a natural felt earthquake $(\mathrm{M}>5)$. The micro seismic waveform contains much higher-frequency content than the earthquake waveform. Larger earthquakes have rupture processes of greater duration (tens of seconds to minutes), which tends to suppress higher-frequency components (e.g., Haskell fault model). The corresponding cutoff frequency or corner frequency is generally inversely proportional to earthquake size. Micro seismic data are characterized by smaller magnitudes, higher frequencies, shorter wavelengths, and shorter duration. Conversely, most felt earthquake data are characterized by larger magnitudes, lower frequencies, longer wavelengths, and longer duration. Based on scaling relationships between various source parameters (including frequency content and magnitude), processing techniques developed for natural felt earthquakes have been applied to micro seismic data. Micro seismic monitoring is an important tool for monitoring fluid flow and pressure/stress changes in the subsurface during fluid injection and extraction, especially during hydraulic fracturing for enhanced hydrocarbon production and geothermal energy production. In general, we record the seismic waves generated by micro seismic events with geophone arrays at or near the earth's surface and in boreholes. Because we cannot predict the exact sourceexcitation times of micro seismic events, we measure ground motion continuously and try to detect micro seismic waveforms in the continuous recorded data. For all micro seismic events detected, we locate the (x, y, z) spatial location (hypocenter) and the $\mathrm{Mw}<0$ induced micro seismic event (blue) and an $\mathrm{Mw}>5$ natural felt earthquake (red). [14]

Kai et al (2014) utilized from particle swarm optimization algorithm (an algorithm of data mining) to forecast seismic events. They applied forecasting process on the seismic database and created a significant improvement (Cai and et al, 2014) [15], Landerb et al (2015) investigated the relationship between sea depth, volcano and earthquake using geophysical approaches of data mining and proposed a method to forecast the occurrence of these seismic events [16]. Mark Last et al (2016) emphasized on forecasting seismic events in Israel and its neighbors. They conducted their predictions by combining time series methods and data mining approaches (Land grebe and R. D. Muller, 2015) [17]. Inshuman et al (2017) utilized from a model named MOIDIS to estimate and forecast seismicity on the ice surface [18]. They focused on the experiments of 2015 in their study (Anshuman and et al, 2017) [19].

\subsection{Other's Applied Works}

The classification relies on differences in signal energy distribution between natural and artificial seismic sources. Jari Kortström and his colleagues have filtered seismic records via 20 narrow band-pass filters and divided them into four phase windows: P, P coda, S, and S coda. They then computed a short-term average (STA) value for each filter channel and phase window. The 80 discrimination parameters served as a training model for the SVM. They calculated station specific SVM models for 19 on-line seismic stations in Finland. The training data set included 918 positive (earthquake) and 3469 negative (no earthquake) examples. An independent test period determined method and rules for integrating station specific classification results into network results. Finally, they applied the network classification rules to independent evaluation data comprising 5435 fully automatic event determinations, 5404 of which had been manually identified as explosions or noise, and 31 as earthquakes. The SVM method correctly identified $94 \%$ of the non-earthquakes and all but one of the earthquakes. The result implies that the SVM tool can identify and filter out blasts and spurious events from fully automatic event solutions with a high level of accuracy. The tool helps to reduce the work-load and costs of manual seismic analysis by leaving only a small fraction of automatic event determinations, the probable earthquakes, for more detailed seismological analysis. The self-learning approach presented here is flexible and easily adjustable to the requirements of a denser or wider high-frequency network. [20].

Based on latest achievements Data Science specialists, SVM, KNN, C4.5, MLP are from most important and popular and leading classifiers in the data specialist world society. It's visible in recent reports of Data Mining international 
institutions such as Rexer-KDnugget-Gartner and also newest authentic articles that mentioned in references. No need to less that one of the most important step in data mining process is correct event detection and data classification. In this paper improvement of data detection and classification has been shown usage a modern method by Boost Learning System.

\section{Data Gathering and Analysis}

In present study, the limited experimental seismic method has been used to carry out related experiments. The used tools and devices are as follow:

- The SPseise3 seismograph with sensors (geophones) connected to the device at a distance of 2.5 meters from each other.

- A calibrated $20 \mathrm{Kg}$ weight.

- Calibrated meter.

The environmental conditions of experiment included a natural environment in a land with rugged soil and asymmetric geometry and in three steps as follow:

- In an area without slope

- In an area with positive slope: in this step, the sensor is located in a point higher than landing level of the weight.

- In an area with negative slope: in this step, the sensor is located in a point lower than landing level of the weight.

The experiment was carried out by creating hit and shake on the ground through leaving $20 \mathrm{~kg}$ weight from heights of $0.5 \mathrm{~m}, 1 \mathrm{~m}$ and $1.5 \mathrm{~m}$, in a way that three times falling was performed from aforementioned heights in each of the three surfaces (without slope, positive slope and negative slope). Since three steps of experiment were carried out each of the surfaces and data were received from three sensors in each step, the total of obtained data equals to $3 * 3 * 3=27$ which a sample of extracted signals has been shown in this section in diagrams.

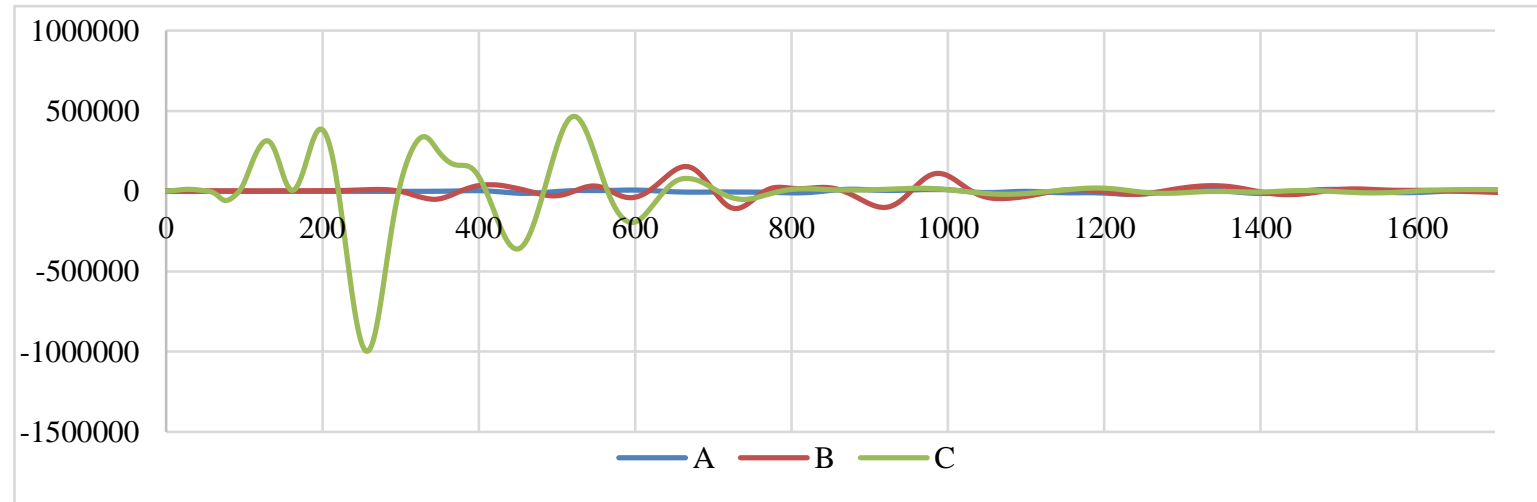

Figure 1. Falling from a height of $0.5 \mathrm{~m}$ on a non-sloping surface

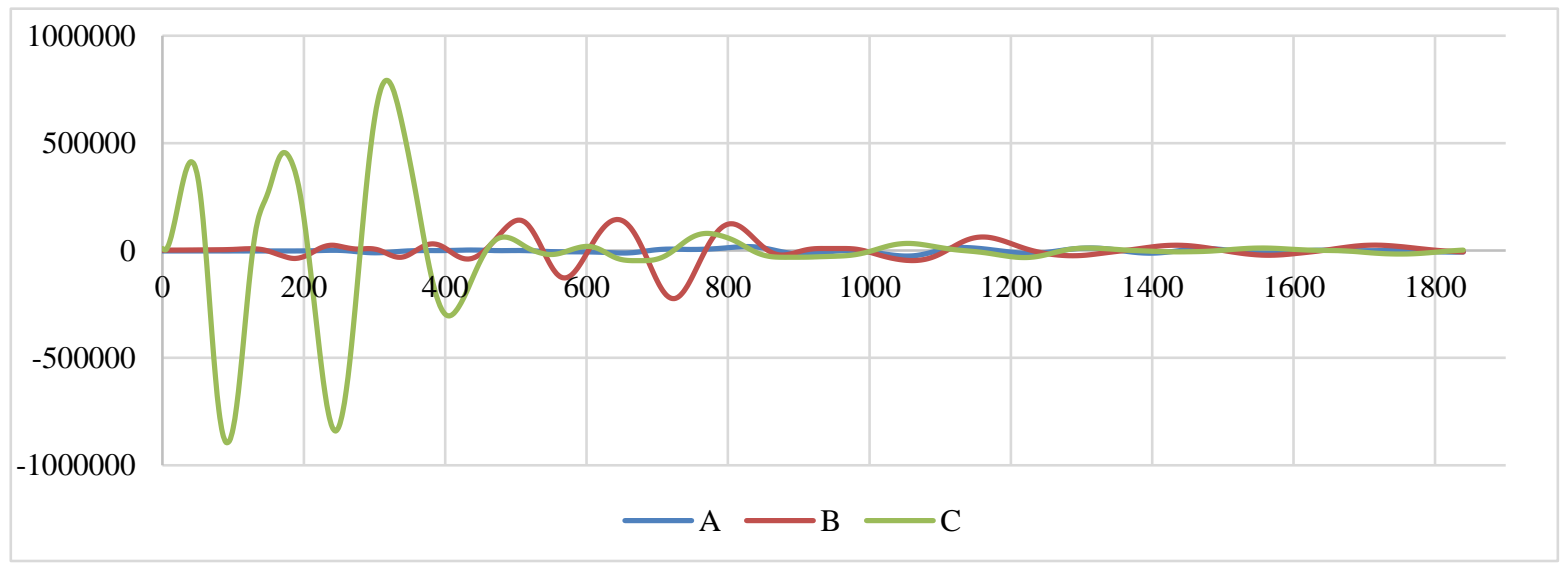

Figure 2. Falling from a height of $1 \mathrm{~m}$ on a non-sloping surface 


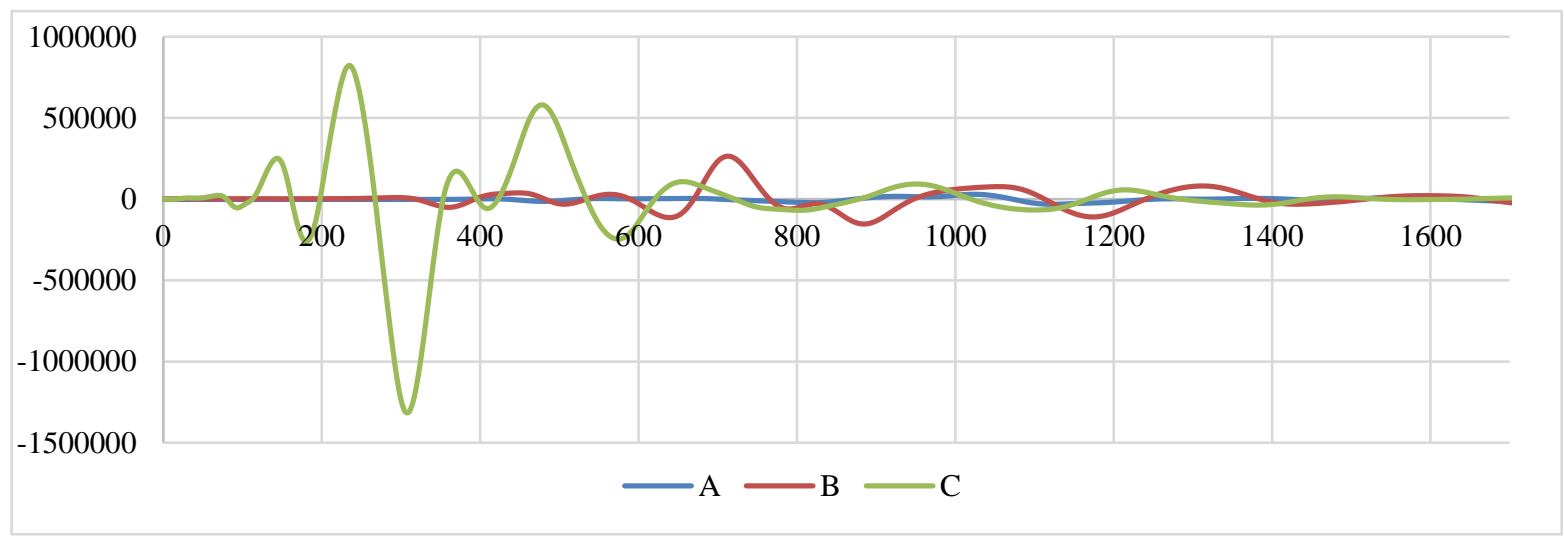

Figure 3. Falling from a height of $1.5 \mathrm{~m}$ on a non-sloping surface

After carrying out related experiments, a set of features was extracted which the features were used to evaluate and conduct related algorithms. Table 1. presents the features have been used in present study.

\subsection{Average Velocity of p-wave}

In this experiment, the average velocity of the initial wave propagation, which is important for analysis, is $500 \mathrm{~m} /$ $\mathrm{s}$, which is obtained by dividing the distance between the sensor and the hypocentre (seismic focus) by the time that the signal is received by the device.

\subsection{Sample Rate}

In this research, discrete sampling was performed on continuous wave with the time period of 267 microseconds. The numbers in the Excel data sheet are the wave amplitude in micro-volt, i.e. in every 267 microseconds, a point of the signal is read. In the following tables, examples of datasets are presented.

\subsection{The Sample of Datasets}

Table 1. Dataset 1

\begin{tabular}{lccc}
\hline & \multicolumn{3}{c}{ No slope } \\
\hline Height from earth surface & $0.5 \mathrm{~m}$ & $1 \mathrm{~m}$ & $1.5 \mathrm{~m}$ \\
Sensor1-2.5m to hit point & $\underline{470}$ records & $\underline{470}$ records & $\underline{470}$ records \\
Sensor2-5m to hit point & $\underline{470}$ records & $\underline{470}$ records & $\underline{470}$ records \\
Sensor3-7.5m to hit point & $\underline{470}$ records & $\underline{470}$ records & $\underline{470}$ records \\
\hline
\end{tabular}

Table 2. Dataset 2

\begin{tabular}{cccc}
\hline & \multicolumn{3}{c}{ Positive slope 10\% } \\
\hline Height from earth surface & $0.5 \mathrm{~m}$ & $1 \mathrm{~m}$ & $1.5 \mathrm{~m}$ \\
Sensor1-2.5m to hit point & $\underline{470}$ records & $\underline{470}$ records & $\underline{470}$ records \\
Sensor2-5m to hit point & $\underline{470}$ records & $\underline{470}$ records & $\underline{470}$ records \\
Sensor3-7.5m to hit point & $\underline{470}$ records & $\underline{470}$ records & $\underline{470}$ records \\
\hline
\end{tabular}

Table 3. Dataset 3

\begin{tabular}{cccc}
\hline & \multicolumn{3}{c}{ Negative slope 10\% } \\
\hline Height from earth surface & $0.5 \mathrm{~m}$ & $1 \mathrm{~m}$ & $1.5 \mathrm{~m}$ \\
Sensor1-2.5m to hit point & $\underline{470}$ records & $\underline{470}$ records & $\underline{470}$ records \\
Sensor2-5m to hit point & $\underline{470}$ records & $\underline{470}$ records & $\underline{470}$ records \\
Sensor3-7.5m to hit point & $\underline{470}$ records & $\underline{470}$ records & $\underline{470}$ records \\
\hline
\end{tabular}


Technical specifications of SPsies-3

Number of recording channels: $4(3+1$ AUX)

Frequency recording range: $5-4600 \mathrm{~Hz}$

Analog anti-aliasing filter options: $250,500,1000 \mathrm{~Hz}$

Sampling interval options: 32, 64, 125, 250, 500, 1000 or $2000 \mu \mathrm{s}$

Analog to Digital resolution: $24 \mathrm{~b}$ it

Hardware gain options: $24,36,48,60 \mathrm{~dB}$

Maximum recording g data length: Up to 16384 samples

Pre -trigger delay: Up to 4096 samples

Table 4. presents the features have been used in present study. In this table sensors has been separated numbered for better diagnosis in different situation.

Table 4. Data Features

\begin{tabular}{|c|c|c|c|}
\hline & \multicolumn{3}{|c|}{ Height $=0.5$ m (II) } \\
\hline & $A($ far $)$ & B (middle) & $\mathrm{C}$ (near) \\
\hline without slope & s7 & s8 & s9 \\
\hline positive slope & $\mathrm{s} 10$ & s11 & $\mathrm{s} 12$ \\
\hline \multirow[t]{3}{*}{ negative slope } & s19 & $\mathrm{s} 20$ & $\mathrm{~s} 21$ \\
\hline & \multicolumn{3}{|c|}{ Height = $1.0 \mathrm{~m}$ (II) } \\
\hline & $A($ far) & B (middle) & C (near) \\
\hline without slope & s1 & s2 & s3 \\
\hline positive slope & s16 & s17 & s18 \\
\hline \multirow[t]{3}{*}{ negative slope } & $\mathrm{s} 22$ & s23 & s24 \\
\hline & \multicolumn{3}{|c|}{ Height = $1.5 \mathrm{~m}$ (II) } \\
\hline & $A($ far $)$ & B (middle) & C (near) \\
\hline without slope & s4 & s5 & s6 \\
\hline positive slope & s13 & s14 & s15 \\
\hline negative slope & s25 & s26 & s27 \\
\hline
\end{tabular}

In Table 4, the columns related to t height indicate the heights of weight falling and the rows are for the slope of surface. S1 indicates positive slope and means the down to the top route. S2 indicates negative slope and meant the top to the down route and S3 indicates flat and non-slope surface. The columns of the second row indicate three sensors have been used in the experiment. Sensor A is the farthest sensor from the source $(7.5 \mathrm{~m})$, sensor B is the middle one from the source $(5 \mathrm{~m})$ and sensor $\mathrm{C}$ is the nearest sensor to the source $(2.5 \mathrm{~m})$. For example, $\mathrm{S} 7$ indicates the received signal from sensor (A) i.e. the farthest sensor from the source in the first falling i.e. from the height of $0.5 \mathrm{~m}$ in nonslope surface. The rest of the experiment is in a same way, i.e. S27 indicates the signal received from the nearest sensor to the source (sensor $\mathrm{C}$ ) in falling from the height of $1.5 \mathrm{~m}$ in the negative slope surface (down to top route). Different statuses of the experiment with different heights and sensors have been shown in following figure.

After gathering data from different sensors in far, middle and near distances as well as in different statuses of nonslope, positive slope and negative slope, it was needed to combine data and produce a coherent dataset to determine the cluster of each sample. For this purpose, all of the gathered data were prepared in the form of Excel file. Therefore, in present study, the experiments were carried out on 12690 samples.

\section{Description of the Proposed Method}

In this section, the process of seismic events detection and classification has been firstly described and then, the learning algorithms and how to produce related models have been investigated. Finally, methods' combination and 
evaluating criteria have been described.

\subsection{Seismic Events Classification}

Nowadays, Software and data mining tools like Rapid-Miner have provided opportunities to apply machine learning algorithms on a set of data and extract variety of outputs. In present study, data were firstly gathered and then required pre-processing acts were applied on the seismic events data and a coherent dataset was produced using Rapid-Miner software and machine learning algorithms including SVM algorithm, MLP neural network algorithm, and C4.5 decisionmaking tree and KNN algorithm. The produced dataset was used to create related model based on the training samples. Training data involved $80 \%$ of micro seismic event samples and were used to training process of model of mentioned algorithms. The experimental data involved $20 \%$ of micro seismic event samples and were used to evaluate the proposed method and each of the mentioned algorithms. The sampling method of experimental data was based on the balancing technique (balance sampling technique). It would be possible to extract the same samples from each of the groups by this technique to cover all of the groups. After producing coherent data set from a set of recorded seismic events and separating training data from experimental data, the data were inserted to the core of learning machine algorithms. The following figure represents how insert data, producing training models and finally classification of each sample by learning machine algorithms.

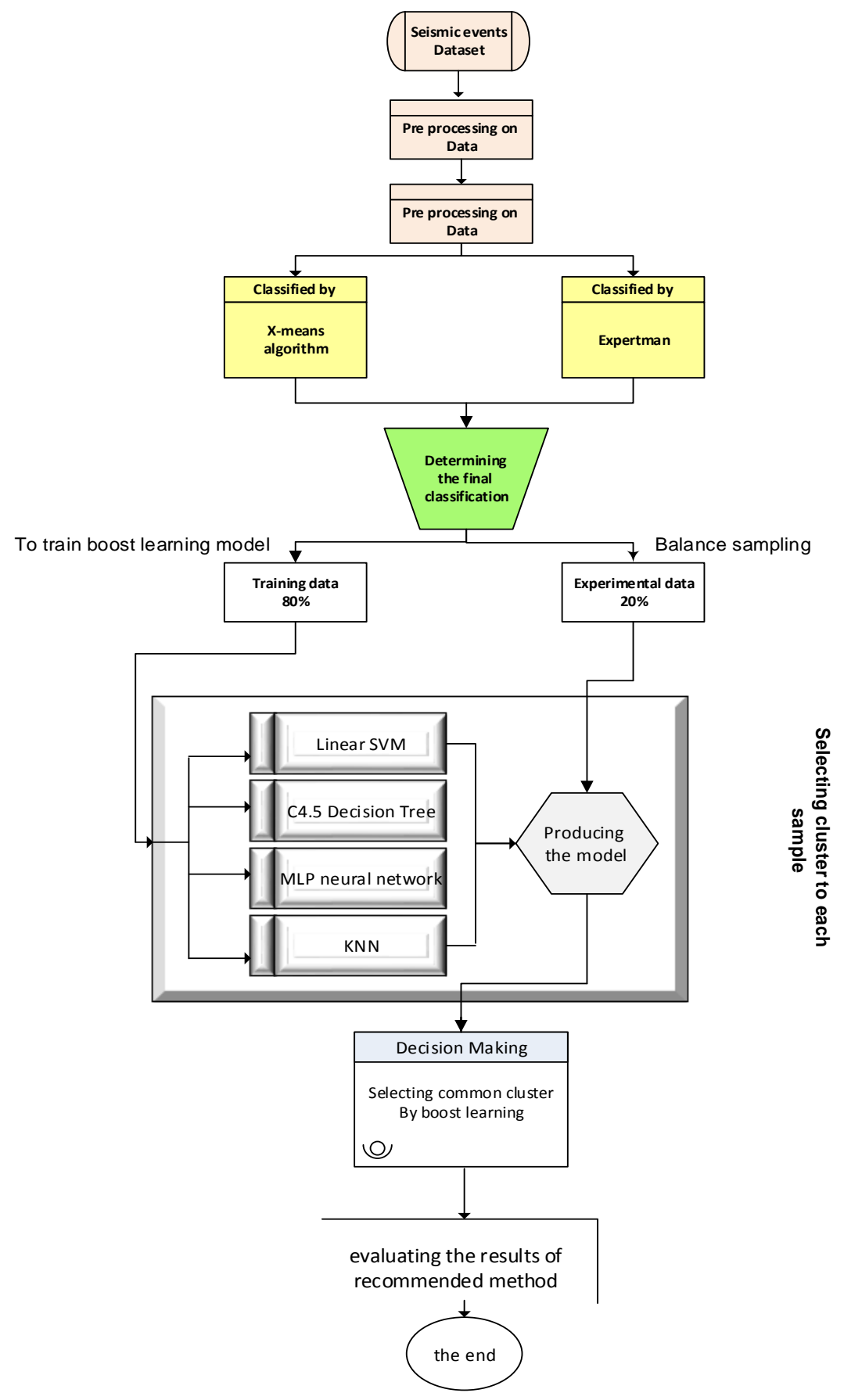

Figure 4. Applying machine learning algorithms on micro seismic events 
As it can be seen from Figure 5, all seismic events recorded by seismograph machine and sensors were firstly inserted to the proposed system as inputs. Then, the data were gathered in a unique profile and turned into a set of coherent events. The classification process was performed by an expert based on the less important and important events and so on. In present study, the X-means clustering algorithm was used to measure the accuracy of classification done by expert as well as to determine cluster for all samples and it was observed that classes are exactly as same as clusters. Therefore, the classes determined by the expert were reliable. Data were separated in two groups of experimental and training after producing a coherent data set and classifying them by expert and X-means algorithm. Then, the training data were inserted to each of the algorithms one-by-one. Each algorithm produced the related model to classify experimental samples by receiving training data. Then, each classifier algorithm sent a cluster of each sample to the input of boost learning algorithm.

\subsection{Boost Learning System}

The structure of boost learning algorithm is in a way that the out puts of algorithms of support vector machine learning with linear regression, MLP Neural Network, C4.5 decision-making tree and KNN receive a cluster and determine the number of which cluster is more than other clusters. The cluster with the most candidates for each sample is selected as a final output. The flowchart of boost learning has been shown in Figure 6.

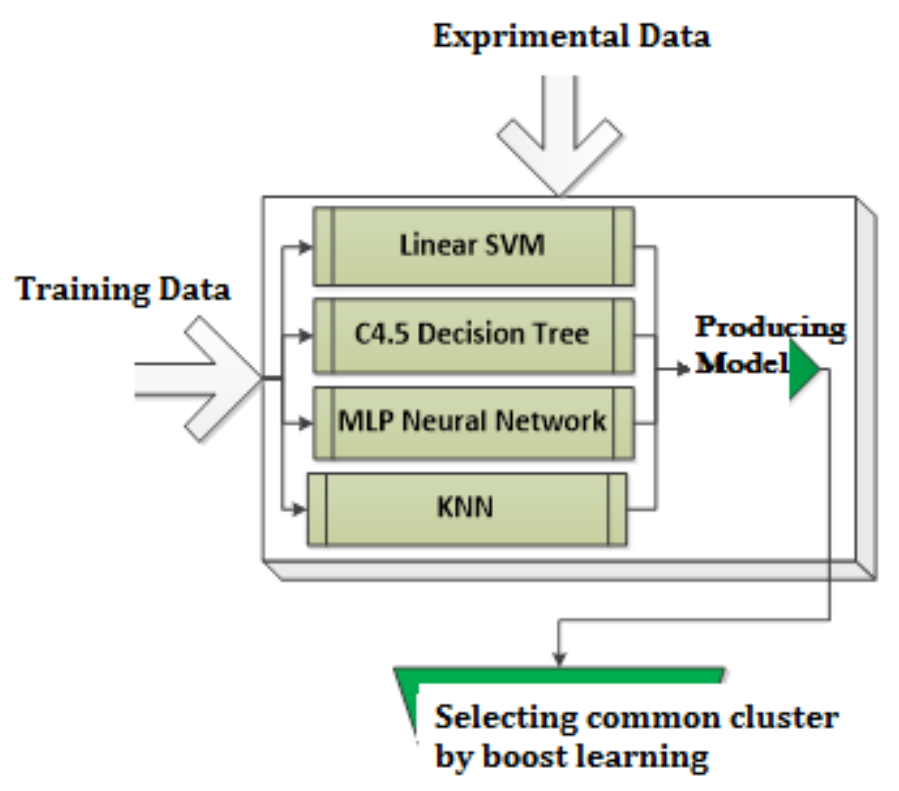

Figure 5. Flowchart and architecture of boost learning method

As it can be seen from Figure 6, a cluster is sent to output of each seismic event sample per repetition as the optimum cluster. The optimum cluster among the output of algorithms can be selected using following equation.

$$
B_{i}=\max \left(C_{1} ; C_{2} ; C_{3} ; \ldots ; C_{n}\right)
$$

Where, $\mathrm{Bi}$ indicates the final cluster to $\mathrm{i}^{\text {th }}$ sample and $\mathrm{C}$ is the determined cluster to $\mathrm{h}^{\text {th }}$ cluster by deep learning algorithms.

\subsection{Evaluation Criteria}

In all of classifier and forecaster algorithms, the accuracy, precision, recalling and real error are the most criteria of evaluation. The following equation presents how to calculate the accuracy of seismic events' classification.

$$
\text { Accuracy }=\frac{T P+T N}{T P+T N+F P+F N}
$$

Where, TP indicates the number of detections and classifications which have been classified as less important or important. TN indicates the number of detections and classifications which have been classified as non-important and FP is for false classifications. The following equation shows how to calculate the recalling of seismic events' classification.

$$
\text { ReCall }=\frac{T P}{T P+F N}
$$

The following equation shows how to calculate the precision of seismic events' classification. 


$$
\text { precision }=\frac{T P}{T P+F P}
$$

The following equation shows how to calculate the error rate of seismic events' classification.

$$
\text { Error }=100-\frac{T P+T N}{T P+T N+F P+F N}
$$

The following equations show how to calculate the mean of real errors and mean square of errors.

$$
\begin{aligned}
& M S E=\operatorname{Mean}\left(\text { Error }^{2}\right) \\
& R M S E=S q r(\text { Error })
\end{aligned}
$$

\section{Results evaluation}

In present study, the forecasting and classification process of micro seismic events was carried out on 3330 samples of data recorded by SPseise 3 seismograph machine consisting up of Three sensors (geophones) connected to the machine with a distance of $2.5 \mathrm{~m}$ from each other, a $20 \mathrm{~kg}$ calibrated weight and a calibrated meter. $20 \%$ of data extracted from whole of the data in a balance form were used as experimental data and $80 \%$ of them used as training data in order to evaluate data mining algorithms as well as to combine optimum algorithm in the form of a boost learning system. Therefore, in this section, the results of all algorithms have been analysed and evaluated based on the mentioned evaluation criteria.

Since the samples extracted from available sensors were without monitoring, it was firstly needed to determine a cluster for each sample and then, machine learning algorithms with monitoring include SVM, neural network, decisionmaking tree and so on applied on the final clustered data. In present study, three thresholds have been defined to detect three different classes as follow:

- Data obtained from dropping the weight from a height of half a meter: noise class

- Data obtained from dropping the weight from a height of one meter: less important events class

- Data obtained from dropping the weight from a height of one and a half meters: important events class

The learning algorithms can be applied on data with determining the classes related to each sample. Following figure represents a comparison between the precision of micro seismic event detection and classification of different algorithms including MLP neural network, SVM, C4.5 decision-making tree, KNN and proposed boost method.

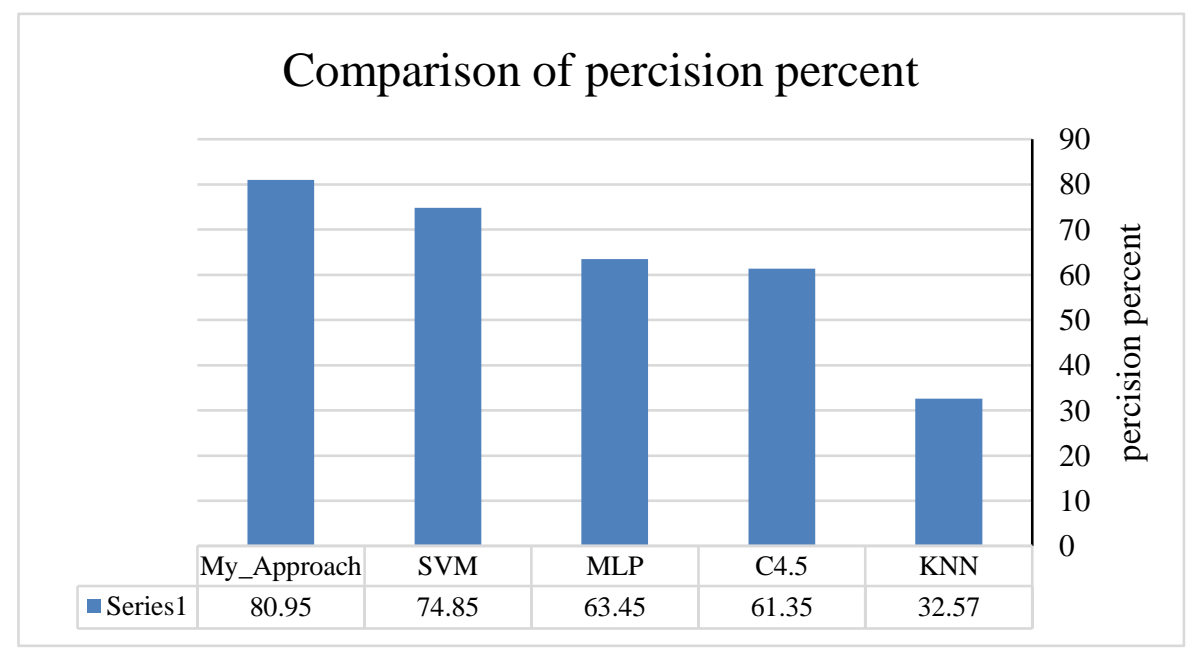

Figure 6. A comparison between precision of micro seismic event detection and classification of proposed method with the other methods

As it can be seen, the level of precision of proposed method to the best answer has been improved up to $6.1 \%$ compare to the other methods. Following figure represents a comparison between the total error rate of micro seismic event detection and classification of different algorithms including MLP neural network, SVM, C4.5 decision-making tree, KNN and proposed boost method. 


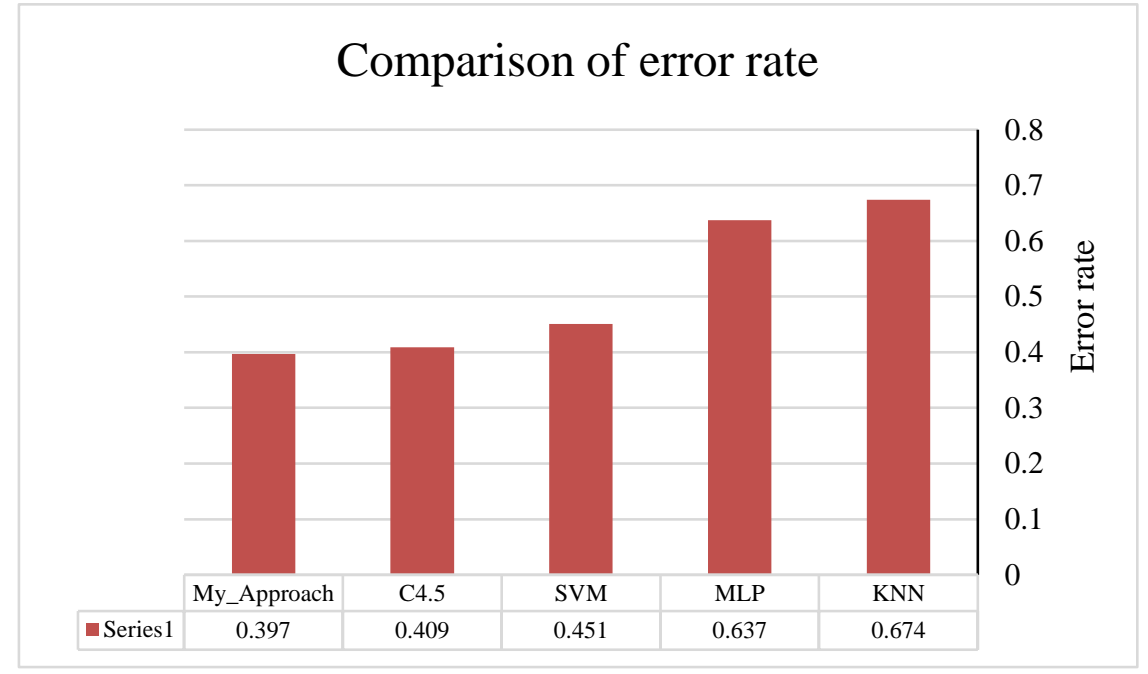

Figure 7. A comparison between error rate of micro seismic event detection and classification of proposed method with the other methods

Since reduction in error rate of proposed method is complementary of precision level, it can be seen that the error rate has been improved up to $0.82 \%$ compare to the other methods. Following figure represents a comparison between the recalling and accuracy of micro seismic event detection and classification of different algorithms including MLP neural network, SVM, C4.5 decision-making tree, KNN and proposed boost method.

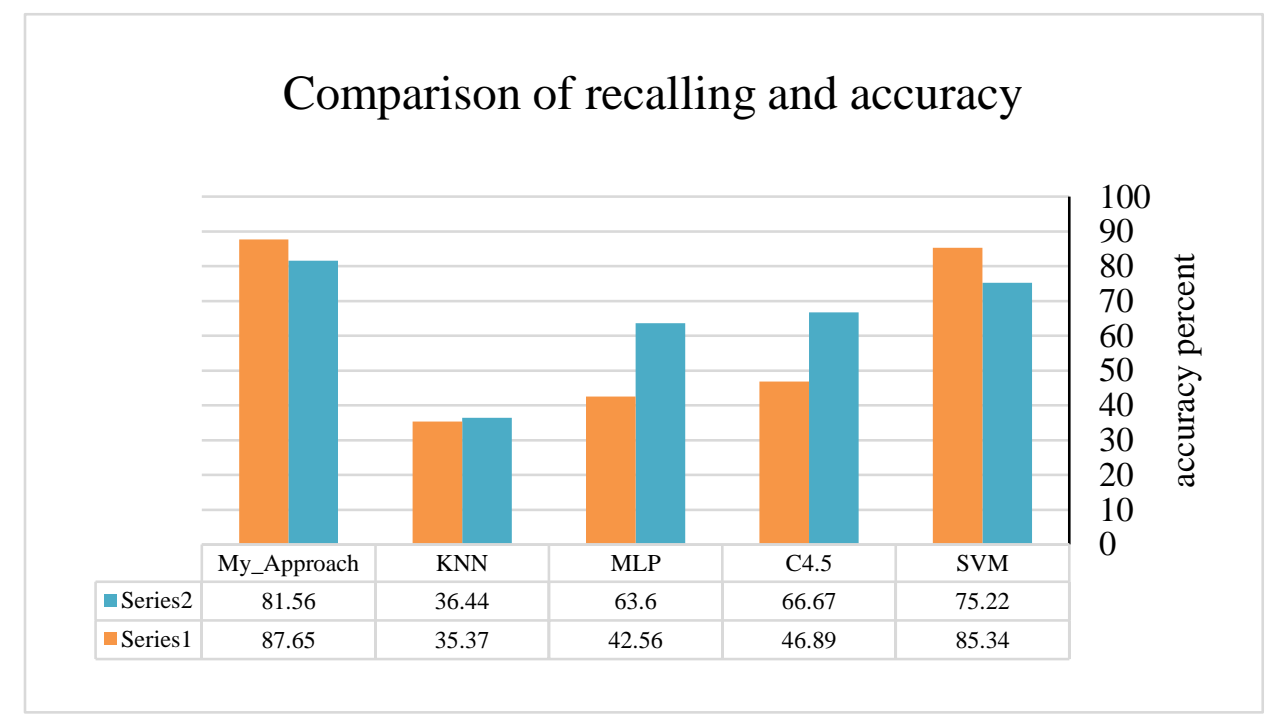

Figure 8. A comparison between recalling and accuracy of micro seismic event detection and classification of proposed method with the other methods

As it can be seen, the level of accuracy and recalling of micro seismic event detection and classification in the proposed method have been improved compare to the mean accuracy of other methods up to $37.4 \%$ and $42.5 \%$, respectively. Following figure represents a comparison between the RMSE values of micro seismic event detection and classification of different algorithms including MLP neural network, SVM, C4.5 decision-making tree, KNN and proposed boost method. 


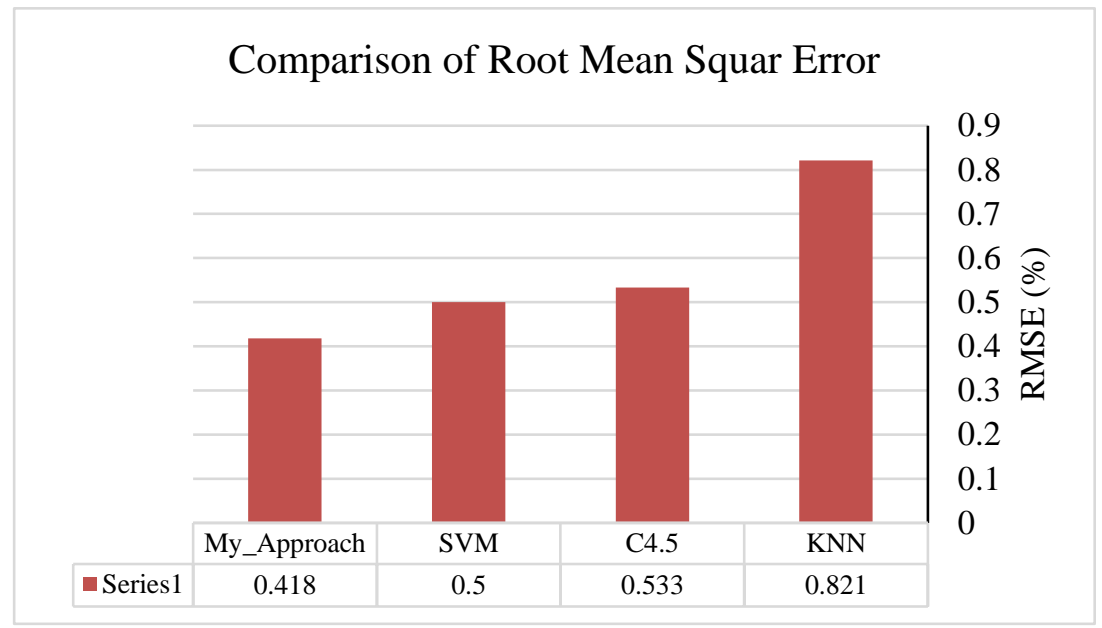

Figure 9. A comparison between RMSE value of micro seismic event detection and classification of proposed method with the other methods

Following figure represents a comparison between the mean real error of micro seismic event detection and classification of different algorithms including MLP neural network, SVM, C4.5 decision-making tree, KNN and proposed boost method.

Finally, with calculating the value of mean error rate and error mean square, it was observed that the proposed boost method detects and classifies the seismic with higher efficiency compare to the other methods.

\section{Conclusion}

In present study, the micro seismic events were detected and classified through combining machine learning methods including back up vector machine, MLP neural network, and C4.5 decision-making tree and KNN algorithm in the form of boost learning. The procedure of experiment was in a way that less important and important seismic events caused by weight falling from various heights and different distances of far, middle and near recorded by laboratory devices and sensors. Then, the data were pre-processed and classified. The classification was based on the level of height, distance and considered sensors. After this step, the training data (80\%) and experimental data $(20 \%)$ were separated from each other. The training data were used to train considered algorithms and the experimental data were used to evaluate the precision, accuracy, and recalling and error rate of the proposed method. In the next step, the algorithms of support vector machine, MLP neural network, C4.5 decision-making tree and KNN produced related models and the new and without class experimental data were inserted to the models in order to evaluations. The cluster related to each sample was determined by each of the algorithms and the answers inserted to the boost learning system. Finally, the cluster with the most number of candidates was selected as the main cluster of related event. The precision and accuracy were significantly improved by this strategy. After simulation of the proposed method, it was observed that the precision of proposed boost method was improved up to $6.1 \%$ compare to the other methods. The error rate improved up to $0.82 \%$ and the recalling and accuracy of detection and classification to the best answer were also improved in the proposed method up to $2.31 \%$ and $6.34 \%$, respectively.

\section{References}

[1] Monadi, A., B. Eslampour, N. Baghaeimehr and F. Abed, Crisis Management in Cities from Holy Quran Perspective, Journal Appl. Environ. Biol. Sci, Vol. 2, No. 12, 2012, pp. 606-608.

[2] Solymani, A., A. Abdolahii and M. Rahimi, Evaluation and Improvement of Urban Worn against Earthquakes (Case Study Neighborhood of Shiraz Zvalanvar), Journal Appl. Environ. Biol. Sci, Vol. 4, No. 1, 2014, pp. 157-165.

[3] Abraham, A., Rule-based expert systems, Handbook of measuring system design, 2005, pp. 909-919.

[4] Hadjimichael, M., A. P. Kuciauskas, P.M. Tag, R.L. Bankert and J.E. Peak, A meteorological fuzzy expert system incorporating subjective user input, Knowl Inf Syst, Vol. 4, No. 3, 2002, pp. 350-369.

[5] Mansiya, K., Z. Alma, M. Torgyn, M. Marzhan and N., Kanat, The methodology of expert systems, International Journal of Computer Science and Network Security, Vol. 14, No. 2, 2014, pp. 62-66.

[6] A. Shahbahrami and Z. Mehdidoust Jalali, Evaluation of Different Data Mining Algorithms to Predict Earthquakes Using Seismic Hazard Data, Journal Appl. Environ. Biol. Sci, Vol. 7, Vol. 2, 2017, pp. 142-150.

[7] Larose, D. T, Discovering knowledge in data: an introduction to data mining, John Wiley \& Sons, 2014. 
[8] Zaki, Mohammed J., Wagner Meira Jr, and Wagner Meira. Data mining and analysis: fundamental concepts and algorithms. Cambridge University Press, 2014.

[9] Suthaharan, Shan. "Support vector machine." In Machine Learning Models and Algorithms for Big Data Classification, pp. 207235. Springer US, 2016.

[10] Zhu, Ji, and Trevor Hastie. "Kernel logistic regression and the import vector machine." Journal of Computational and Graphical Statistics 14, no. 1 (2005): 185-205.

[11] Rui, H. O. U., and Bi-xi Zhang. "A Method for Forecasting Regional Logistics Demand Based on MLP Neural Network and Its Application [J]." Systems Engineering-theory \& Practice 12 (2005): 006.

[12] J. Ross Quinlan, C4.5: Programs for Machine Learning, Computer Science Artificial Intelligence Machine Learning, $2014,302$.

[13] Liu, Z. G., Pan, Q., \& Dezert, J, A new belief-based K-nearest neighbor classification method, Pattern Recognition, Vol. 46, No. 3, 2013, pp. 834-844.

[14] Rie Kamei1, Nori Nakata2, and David Lumley1, Introduction to micro seismic source mechanisms, THE LEADING EDGE August 2015

[15] Entao Liu, Lijun Zhu, Anupama Govinda Raj, James H. McClellan, Abdullatif Al-Shuhail, SanLinn I. Kaka, Naveed Iqbal Micro seismic events enhancement and detection in sensor arrays using autocorrelation based filtering, arXiv:1612.01884 [physics.geo-ph]

[16] S.Z. Cai, Q.F. Zhang, X.P. Xu, D.H. Hu and Y.M. Qu, The Data Mining Technology of Particle Swarm Optimization Algorithm in Earthquake Prediction, Advanced Materials Research, Vol. 9, 2014, pp. 1570-1573.

[17] T. C. W. LANDGREBE AND R. D. MULLER, Uncovering the relationship between sub ducting bathymetric ridges and volcanic chains with significant earthquakes using geophysical data mining, Australian Journal of Earth Sciences, 2015, pp. 162-171.

[18] Mark Last, Nitzan Rabinowitz, Gideon Leonard, Predicting the Maximum Earthquake Magnitude from Seismic Data in Israel and Its Neighboring Countries, Plos One, 2016.

[19] Anshuman Bhardwaj, Shakti man Singhb, Lydia Samb, Akanksha Bhardwajd, Javier Martín-Torresa, Atar Singh, Rajesh Kumar, MODIS-based estimates of strong snow surface temperature anomaly related to high altitude earthquakes of 2015, Remote Sensing of Environment, Vol 188, 2017, pp. 1-8.

[20] Jari Kortström, MarjaUski, TimoTiira, Automatic classification events within a regional seismograph network of seismic Computers \& Geosciences, Volume 87, February 2016, Pages 22-30. 